\title{
(6) OPEN ACCESS \\ Diagnostic procedures for non-small-cell lung cancer (NSCLC): recommendations of the European Expert Group
}

\author{
Manfred Dietel, ${ }^{1}$ Lukas Bubendorf, ${ }^{2}$ Anne-Marie C Dingemans, ${ }^{3}$ Christophe Dooms, ${ }^{4}$ \\ Göran Elmberger, ${ }^{5}$ Rosa Calero García, ${ }^{6}$ Keith M Kerr, ${ }^{7}$ Eric Lim, ${ }^{8}$ \\ Fernando López-Ríos, ${ }^{9}$ Erik Thunnissen, ${ }^{10}$ Paul E Van Schil, ${ }^{11}$ Maximilian von Laffert ${ }^{1}$
}

For numbered affiliations see end of article.

\section{Correspondence to Professor Manfred Dietel, Institute of Pathology, Charité Universitätsmedizin Berlin, Campus Charité, Charitéplatz 1, Berlin 10117, Germany; manfred.dietel@charite.de}

Received 10 December 2014 Revised 10 June 2015 Accepted 21 July 2015 Published Online First 3 November 2015

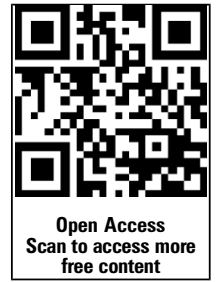

CrossMark

To cite: Dietel $M$,

Bubendorf $L$, Dingemans A-

$\mathrm{MC}$, et al. Thorax

2016;71:177-184.

\section{ABSTRACT}

Background There is currently no Europe-wide consensus on the appropriate preanalytical measures and workflow to optimise procedures for tissue-based molecular testing of non-small-cell lung cancer (NSCLC). To address this, a group of lung cancer experts (see list of authors) convened to discuss and propose standard operating procedures (SOPS) for NSCLC.

Methods Based on earlier meetings and scientific expertise on lung cancer, a multidisciplinary group meeting was aligned. The aim was to include all relevant aspects concerning NSCLC diagnosis. After careful consideration, the following topics were selected and each was reviewed by the experts: surgical resection and sampling; biopsy procedures for analysis; preanalytical and other variables affecting quality of tissue; tissue conservation; testing procedures for epidermal growth factor receptor, anaplastic lymphoma kinase and ROS proto-oncogene 1, receptor tyrosine kinase (ROS1) in lung tissue and cytological specimens; as well as standardised reporting and quality control (QC). Finally, an optimal workflow was described.

Results Suggested optimal procedures and workflows are discussed in detail. The broad consensus was that the complex workflow presented can only be executed effectively by an interdisciplinary approach using a welltrained team.

Conclusions To optimise diagnosis and treatment of patients with NSCLC, it is essential to establish SOPs that are adaptable to the local situation. In addition, a continuous QC system and a local multidisciplinary tumour-type-oriented board are essential.

\section{INTRODUCTION}

The majority of patients with suspected lung cancer require tissue biopsy to confirm the diagnosis. Many patients will present with advanced disease, where mutation testing for targeted treatment is now considered to be the standard of care.

In non-small-cell lung cancer (NSCLC), analysis of epidermal growth factor receptor (EGFR) mutations and anaplastic lymphoma kinase (ALK) inversions/ translocations are prerequisites for determining the appropriate tyrosine kinase inhibitor to be used in targeted treatment in order to improve patient outcomes and survival. ${ }^{1}{ }^{2}$ Besides several other targets that are tested within clinical trials, the ROS protooncogene 1, receptor tyrosine kinase (ROS1) seems to be the third genetic alteration that needs to be implemented in the routine testing procedure. ${ }^{3-5}$
There is no current Europe-wide consensus concerning these aspects. Moreover, a structured recommendation (best practice) and overview for tissue diagnosis and molecular testing in NSCLC is missing. To obtain best results, standard operating procedures (SOPs) are required to optimise clinical sampling, tissue processing, testing, reporting, timing and quality control (QC). In addition, to ensure an adequate workflow, a local interdisciplinary tumour board is absolutely essential. Adherence to best practice in molecular testing of NSCLC is vital to ensure accurate diagnoses and appropriate clinical decisions. Therefore, a European multidisciplinary lung cancer group convened and discussed these above-mentioned aspects. This article summarises their particular statements.

\section{MATERIALS AND METHODS}

To agree recommendations on tissue sampling for diagnosis and molecular testing procedures for NSCLC, a group of European interdisciplinary NSCLC experts convened in Berlin in November 2013. One aim of this activity was to gather information from all physicians involved in the diagnosis and treatment of patients on the importance of the different steps towards optimal diagnosis and treatment. The current paper presents an overview of the essential steps recommended by this group. The experts were selected by MD, ET, KMK and FL-R. The selection procedure was based on a number of earlier meetings involving these individuals when a range of issues relating to NSCLC diagnostics were discussed. These scientifically acknowledged experts in the field were individually invited to the meeting, to present their data and to share their experiences, with a view to producing a consensus publication. At the outset of the meeting in Berlin, the structure of the process and the main topics were discussed and agreed by the group. Each individual participant made a presentation to the group, a broad discussion took place, the main issues were identified and a consensus position was reached. Finally, the experts agreed to write the corresponding section of a consensus manuscript on their particular topic and MD and MvL were asked to merge the different contributions together into a structured, consensus paper. The draft was then circulated several times among the participants for final editing and completion. The meeting was funded by Pfizer (Europe), but the latter had no input into the paper content. 


\section{RESULTS}

Diagnosis, tissue sampling and staging: role of the thoracic surgeon

Most patients with suspected lung cancer require a tissue-based diagnosis. The aims of tissue sampling include confirmation of diagnosis (eg, adenocarcinoma vs squamous cell carcinoma) and molecular testing. ${ }^{6}$ Individual patient care by a multidisciplinary team is best practice to decide individualised diagnostic and therapeutic plans. ${ }^{7}$ Preliminary staging investigations such as CT and positron emission tomography/CT are often helpful to guide invasive sampling. Where possible, the least invasive method of biopsy is undertaken.

Distant metastases, if accessible, may be the first site of puncture or biopsy, as these tissues can provide diagnostic and staging information (proof of M1b disease). Cervical mediastinoscopy, thoracoscopy and related procedures allow biopsies of every mediastinal lymph node station (stations 1-9) or pulmonary parenchymal lesion. These procedures provide large samples, but require general anaesthesia. With the latter two techniques, false-negative rates are below $10 \%$, increasing the sensitivity of the method used. ${ }^{8}{ }^{9}$ In more difficult cases, thoracoscopy can also be used to perform wedge excisions of suspicious lung nodules or take biopsies of suspect pleural lesions which are difficult to reach by another method.

At each assessment centre, a precise workflow for transportation of samples should be optimised, with an established SOP to reduce transportation time to the diagnostic and molecular pathologists.

\section{Summary}

- In patients in whom lung cancer is suspected, a tissue diagnosis should be obtained.

- Each patient's case should be discussed within a multidisciplinary team to provide an individualised diagnostic and therapeutic plan.

- The least invasive method of biopsy is preferred, although in some cases invasive procedures may be necessary.

- A SOP should be established for transportation of tissue samples and biopsies.

\section{Biopsy procedures and sampling for analysis}

Approximately $80 \%$ of patients with NSCLC present or relapse with advanced disease. As described above, these patients need highly qualified diagnostic procedures.

\section{Flexible videobronchoscopy}

Taking histological biopsies is preferred over brushes and cytological washes. When an endobronchial tumour is visible during flexible videobronchoscopy, a diagnostic yield of at least $85 \%$ should be obtained. ${ }^{10}$ A diagnostic yield of $70 \%$ or more should be obtained when modern guidance techniques are used to diagnose a peripheral tumour $>20 \mathrm{~mm}$ in size, invisible during bronchoscopy but in proximity to a patent bronchus. In order to optimise diagnostic yield and allow histopathological tumour subtyping and genotyping, at least five endobronchial/transbronchial forceps biopsies should be obtained. ${ }^{11}{ }^{12}$ An additional five bronchial forceps biopsies should be considered in order to maximise the volume of tissue for NSCLC phenotyping and genotyping. Alternatively, two cryobiopsies may be taken during flexible bronchoscopy. Endobronchial ultrasound (EBUS) with transbronchial needle aspiration using a 21-22 G needle has a diagnostic yield of at least $90 \%$ in enlarged or bulky lymph nodes. Oesophageal ultrasonography is able to reach stations 8 and 9, and can also access the left adrenal gland, left liver lobe and coeliac trunk lymph nodes. At least four needle aspiration passes per target lesion are recommended to provide sufficient tissue for genotyping. ${ }^{13}$

Ideally, the pulmonary physician or radiologist performing the biopsy procedure is aliquoting the different biopsies to the laboratory in individual containers.

\section{Radiology-guided percutaneous biopsy}

CT-guided coaxial core biopsy is preferred over aspiration cytology when possible, as it allows multiple and larger samples to be obtained with a single puncture. Careful case selection and technical considerations are necessary to increase diagnostic yield and avoid unnecessary complications. ${ }^{14}$

A diagnostic yield of at least $90 \%$ should be obtained when the target lesion to biopsy is in proximity to the chest wall and $>15 \mathrm{~mm}$ in size. ${ }^{15}$ At least two core needle biopsies are recommended, using an 18-20 G needle. To maximise the volume of tissue for histological subtyping and genotyping, 3-6 core needle biopsies could be considered as long as the safety of the procedure can be guaranteed. Depending on local expertise and availability of techniques, either a CT or an ultrasound-guided percutaneous core needle biopsy can be performed from the primary tumour or a metastatic site (pleural, liver, bone, adrenal gland, peripheral nodal metastasis, etc). The most frequent complications are pneumothorax and haemorrhage, although these are usually of little concern. Only $1-4 \%$ of pneumothorax cases require tube placement. Air embolism and tumour seeding are extremely rare.

\section{Summary}

- At least five endobronchial/transbronchial forceps biopsies should be taken; to maximise the volume of tissue, an additional five forceps biopsies or two cryobiopsies could be considered.

- At least four EBUS/endoscopic ultrasound needle aspiration passes per target lesion are recommended.

- At least two percutaneous core needle biopsies using an 1820 G needle should be taken; in order to maximise the volume of tissue, 3-6 core needle biopsies could be considered.

\section{Handling of tissue (macroscopy)}

Biopsies are transferred immediately to the labs (institutes of pathology), are formalin-fixed and embedded in paraffin. Prior to fixation and embedding, operative material (eg, lobectomy) is initially handled macroscopically (documentation and gross sectioning) after a standardised protocol. ${ }^{16}$ The hilus and the tissue margin (the latter depending on the operation procedure) need to be taken to tell about the R(esection)-status. The operative material is cut into sections (thickness $5-10 \mathrm{~mm}$ ) and the tumour is measured as this will classify the pT-stadium. ${ }^{17}$ Measuring also includes the distance of the tumour to the resections margins; furthermore, pleura infiltration needs to be documented. A total of 3-5 tumour sections are taken, as well as an additional representative section of non-tumour tissue. Small tumours should be embedded completely.

\section{Preanalytical variables and factors affecting quality of biopsies and surgical samples}

Before the pathologist can process surgical samples and biopsies, preanalytical variables affecting sample quality need to be considered. 
Minimal amount of tissue/cells required for reliable analyses Several immunohistochemical markers may need to be analysed to confirm and subtype NSCLC. ${ }^{18}$ Additional material is required if a range of tests are planned in the context of personalised medicine, using immunohistochemistry (IHC), in situ hybridisation (ISH) or sequencing techniques. For these procedures, sufficient material of good quality is required. ${ }^{19}$ In the majority of cases, especially for patients with advanced lung cancer, diagnostic material might be sparse, containing only a small amount of tumour cells, on which all diagnostic tests must be performed. Small biopsy samples with a small number of tumour cells might only allow diagnosis and classification of tumour type (eg, adenocarcinoma), but additional molecular tests may be compromised.

\section{Preanalytical considerations}

To standardise the work-up of resection material, vacuum preservation might be considered. ${ }^{20}$ Immediately after removal, the material can be preserved by sealing under vacuum in special plastic bags and placing it in a controlled environment $\left(4^{\circ} \mathrm{C}\right)$. It is important that these procedures are conducted in a standardised way.

Warm and cold ischaemia time should be as short as possible. It may be valuable to record ischaemia time, as this may impact on subsequent analyses. This 'time delay' between tissue acquisition and fixation depends on environmental temperature and should be shorter than $30 \mathrm{~min}$. In general, a consecutive fixation period in buffered formalin of 6-48 h before paraffin embedding is recommended (depending on the volume of material). This will permit adequate DNA quality and most IHC-detectable antigens will survive. Although RNA is more labile and deteriorates rapidly, the latest quantitative real-time PCR tests can analyse RNA in formalin-fixed, paraffin-embedded (FFPE) samples (eg, the EndoPredict breast cancer assay). ${ }^{21}$ Other fixatives, such as Hepes-glutamic acid buffer-mediated Organic solvent Protection Effect (HOPE), FineFix and alcohol, did not prove to be superior to buffered formalin and did not find widespread acceptance.
Organisation and optimisation of sectioning

To maximise tissue availability, two strategies may be employed. The first is to perform minimally invasive sectioning for an initial look and a preliminary diagnosis - a 'touch and go' approach. This differs significantly from the standard technique practised by most technicians, who are trained to cut deep into the block to make sure the largest diameter of the biopsy is on the initial slides. The other strategy is to cut multiple (approximately 20) unstained sections and keep them stored until a preliminary diagnosis has been made and ancillary testing is requested. Cutting should only be done by the most experienced technicians, using microtomes equipped with 'waterfall' slides to make use of every section. Ultra-thin sections (approximately $2 \mu \mathrm{m}$ ) are excellent for routine staining and IHC. Finally, the frequency of re-cutting blocks should be minimised-ideally, sections for all ancillary tests should be cut in the same session.

To achieve high-quality molecular testing, it is important that the pathologist marks the most suitable tumour area in the slide so that the optimal tumour content is extracted from the paraffin-embedded material. ${ }^{5}$ The most adequate procedure appears to be manual microdissection (figure 1). If several tissue blocks are available, the tumour area with the least amount of necrosis, blood, mucous or inflammation should be selected. The quantitative relationship of tumour cells to non-tumour cells is also of critical importance; if possible, a minimum of $20-30 \%$ of tumour cells should be present in material tested for genetic alterations to minimise false-negative results.

\section{Practical suggestions}

Standardised algorithms for the diagnostic procedures should be defined in current routine practice. ${ }^{22}$ This should involve reflex sectioning for IHC and/or molecular testing, which will shorten turnaround time and preserve tissue (see figure 2). ${ }^{23}$ The first set of slides is required for H\&E, PAS and IHC characterisation for TTF1 and p40 or p63, complemented by CK5/6, napsin or CK7 if necessary. First of all, this will ensure the diagnosis and
Figure 1 Importance of manual microdissection as a prerequisite for reliable and reproducible analyses in molecular pathology. (A-D) A typical lung specimen with five biopsies, of which one contained malignant cells; only this biopsy should be used for molecular analyses. The tumour area must therefore be primarily prepared microscopically from the paraffin block before being analysed. (E) Further analytical steps. (F) Example of a pathology report combining morphological and molecular results as a prerequisite for treatment of a patient with a targeted drug. (G) All tests should be accompanied by external quality assurance, such as 'Qualitätssicherungs-Initiative Pathologie' (QuIP).

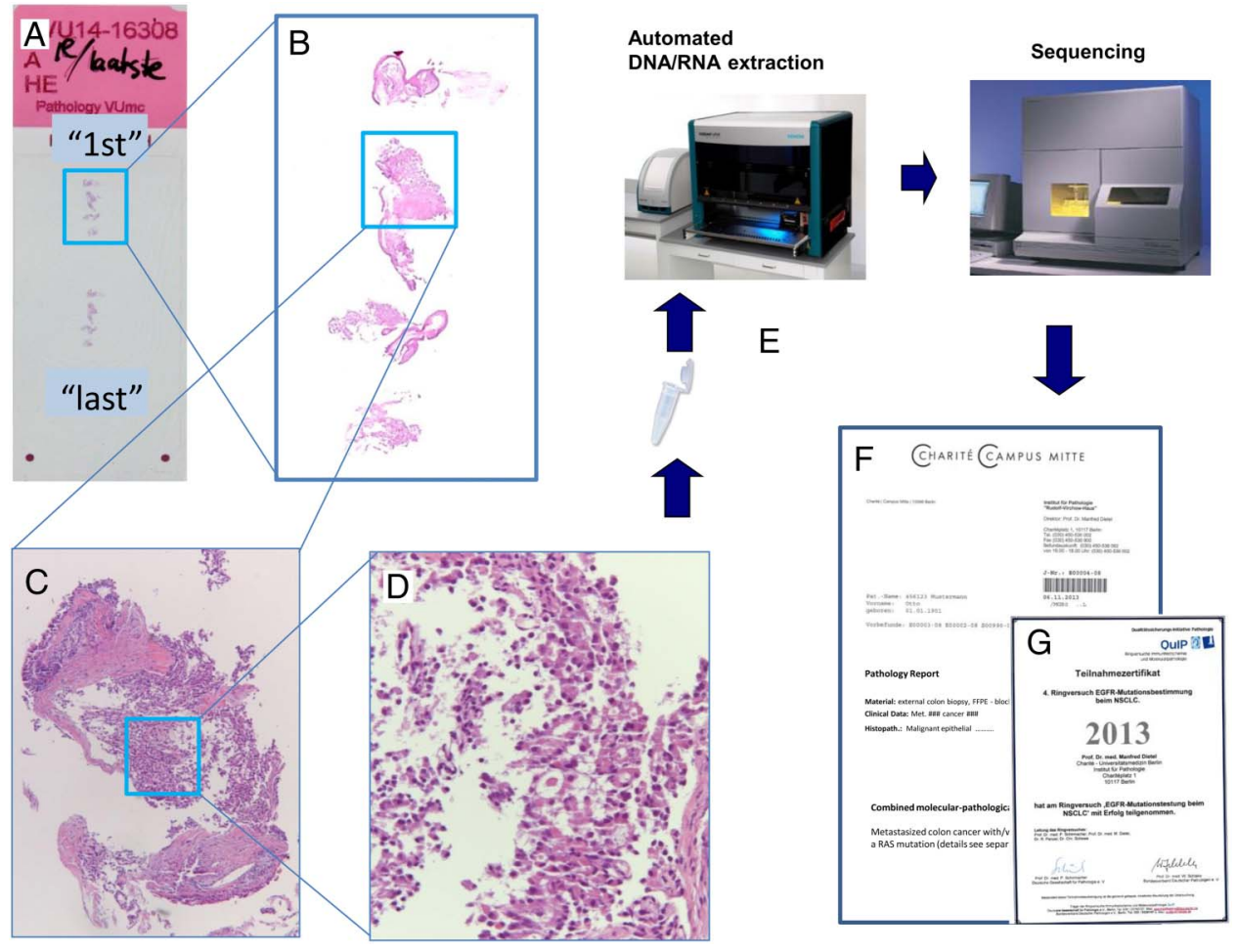


helps to differentiate between adenocarcinoma and squamous cell carcinoma (or not otherwise specified). Parallel predictive IHC (eg, ALK, c-MET, ROS) might be performed. Genetic analyses for EGFR and fluorescent in situ hybridisation (FISH) for ALK are considered routine steps. The analyses of RAS, BRAF, MET, ROS and RET can be considered, if clinically appropriate. Especially ROS1 is more and more requested for testing due to promising study results. So far, FISH seems to be the gold standard, IHC is possible but not well standardised. ${ }^{24}$ Since most of these mutations are mutually exclusive, a pragmatic approach would be to perform sequential testing. However, this is time-consuming, and co-mutations were observed indicating intrinsic or acquired therapy resistance.

\section{Summary}

- Optimise transfer of tissues from operating theatre to pathology laboratory.

- Perform appropriate fixation as early as possible (between 6 and $48 \mathrm{~h})$.

- Aliquoting of biopsy tissue to individual containers and blocks.

- Cut extra sections at the first cutting session to avoid tissue waste, especially if the amount of tissue is low.
- Define parallel IHC-staining to shorten turnaround times (5-10 days), saving tissue and reducing costs.

- Use of controlled, tissue-conserving tumour cell enrichment techniques supervised by an experienced pathologist to select the most appropriate tumour area (manual microdissection) for DNA extraction and molecular testing.

\section{Testing procedures for detection of EGFR and ALK status in lung tissue}

Mutations in the EGFR gene occur in the intracellular domain, in particular the tyrosine kinase domain, in approximately $7 \%$ of resected NSCLC cases and 13\% of adenocarcinomas. ${ }^{25}$ Deletions in exon 19 and the L858R point mutation in exon 21 are the most frequent mutations. The appropriate methods to detect EGFR mutations are Sanger, pyrosequencing and nextgeneration sequencing (NGS) (platform dependent).

Several methods for detecting ALK gene rearrangements are available, including FISH, IHC, reverse transcriptase (RT)-PCR and NGS. ${ }^{19}$ In the USA, ALK FISH is the method of choice, while in Europe, approval for ALK-positive lung cancer also allows other ALK validated tests. ${ }^{19}$

Currently, three antibodies, ALK1, 5A4 and D5F3, have been tested for ALK IHC-positive lung cancer, with 5A4 and D5F3

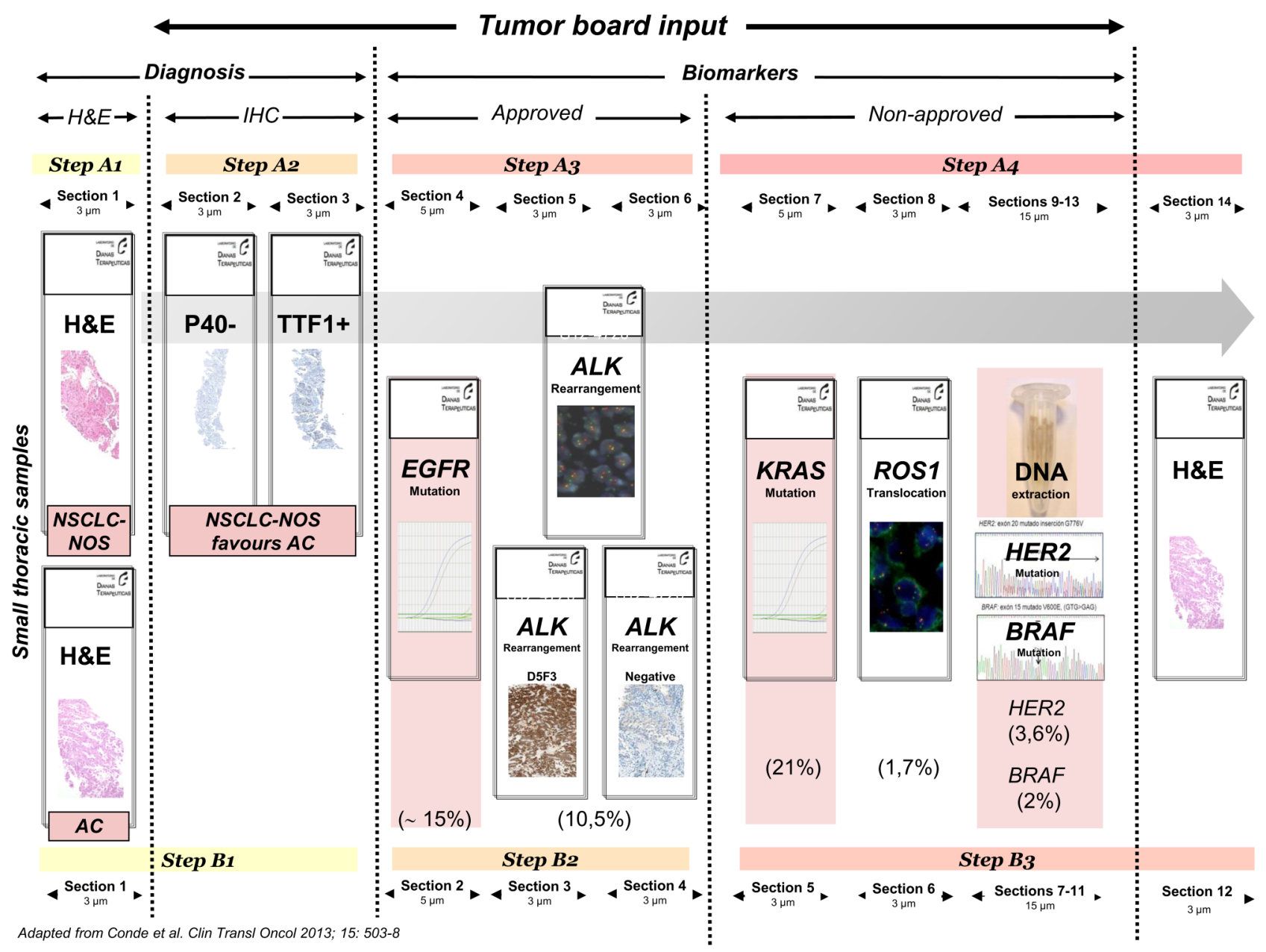

Figure 2 A realistic approach for sample prioritisation for the study of predictive biomarkers in patients with advanced lung adenocarcinomas. Route $A$ is for cases that require classificatory immunohistochemistry (IHC), while route B is for cases that are diagnosed based on H\&E staining alone. The relative frequency of the different genetic alterations is shown in parentheses. Adapted from Conde et al, ${ }^{23}$ under the Creative Commons Attribution licence (CC BY). AC, adenocarcinoma; ALK, anaplastic lymphoma kinase; BRAF, v-Raf murine sarcoma viral oncogene homologue B1; EGFR, epidermal growth factor receptor; HER, human epidermal growth factor receptor; KRAS, V-Ki-ras2 Kirsten rat sarcoma viral oncogene homologue; NSCLC-NOS, non-small-cell lung cancer-not otherwise specified; ROS1, ROS proto-oncogene 1, receptor tyrosine kinase. 
providing the best results. ${ }^{19}$ There is a high correlation between FISH and IHC, although some discrepant cases have been reported. $^{19} 26$

There are two different RT-PCR approaches for ALK testing. One uses probes for both fusion genes (ALK and EML4/KIF5B/ HIP133), ${ }^{27-30}$ while the other compares different levels of amplification of small PCR products $\left(5^{\prime}\right.$ and $3^{\prime}$ portions of ALK transcripts) on the ALK gene (fusion partner independent). ${ }^{31} 32$ The first approach has a sensitivity of approximately $90 \%$ (depending on the coverage of fusion partners), while theoretically the latter approach has $100 \%$ sensitivity. Different mechanisms for resistance exist in lung cancer with ALK gene rearrangement, demonstrating the need for tissue sampling before making new treatment decisions. ${ }^{33} 34$

\section{Summary}

- EGFR and ALK status should be determined in locally advanced/metastasised lung adenocarcinomas for prediction and treatment.

- In recurrent tumours, and tumours treated with EGFR-targeted or ALK-targeted therapies, newly growing lesions should be re-biopsied and tested to determine mechanisms of resistance, possibly uncovering other treatment options.

\section{ALK analysis in cytological specimens}

As many as $40 \%$ of all lung cancers are diagnosed by cytology without concurrent biopsy material, necessitating predictive marker testing of cytological specimens. Initial concerns and prejudices regarding cytological predictive marker testing in lung cancer have disappeared and it is now widely recognised that cytological specimens are suitable for PCR-based or FISH-based predictive marker analyses. Cytological diagnosis is explicitly referred to in recommendations. ${ }^{2} 1819$

\section{Cytology procedures and preanalytics}

Cytological diagnosis of lung cancer is typically based on EBUS-fine needle aspiration (FNA), bronchial cytology, pleural effusions and FNA from distant metastases. The presence of a cytopathologist or a trained cytotechnician during the procedure of EBUS-FNA has become a standard in some institutions in order to ensure an appropriate amount of tumour cells in the sample. FFPE cell blocks are the preferred method for processing cytological specimens in many laboratories, as they can be handled like histological specimens and provide long-term preservation of proteins. However, cell blocks are not always available and a significant subset of cell blocks contain insufficient cancer cells for molecular analysis. ${ }^{35}$ In addition, differentiating tumour cells from adjacent reactive cells in cell blocks is more challenging than in conventional cytology, especially during FISH analysis. In air-dried or alcohol-fixed cytological specimens, DNA quality is better than that of formaldehyde fixation, which leads to crosslinking and chemical modification of nucleotides. This explains the high success rate (close to 100\%) for ALK FISH analysis in conventional cytology and a failure rate of up to $19 \%$ for histological specimens reported by Savic et al. ${ }^{36}$

\section{ALK FISH analysis in cytology}

FISH is a robust technology applicable to almost all types and formats of cytological specimens, including conventional smears, cytospins or liquid-based preparations. The use of adhesive-coated and positively charged slides is recommended to improve the adherence of the cells and prevent them from floating off during technical FISH procedures. FISH works equally well on unstained specimens as well as those stained with Papanicolaou, H\&E or May-Grünwald-Giemsa; a respective protocol has been published. ${ }^{2}$

Precise relocation of tumour cells using an automated stage greatly facilitates FISH scoring and review, especially in cases with a low proportion of tumour cells on the slide. ${ }^{26}$

\section{ALK IHC in cytology}

ALK IHC is a promising method for preselecting cytological specimens for FISH testing, and may even remove the need for FISH analysis. In a recent study of ALK IHC using the 5A4 antibody, the accuracy of ALK detection on Papanicolaou-stained cytological slides was high, with a sensitivity and specificity of almost $100 \%$ compared with ALK FISH. ${ }^{37}$ ALK IHC of cytological specimens also works with other appropriate antibodies (eg, D5F3) and immunostainers, provided the appropriate protocols are being developed. For cell blocks, existing protocols and assays for ALK IHC of histological specimens can be applied.

\section{Summary}

- Histological and cytological specimens should be reviewed jointly to select the most appropriate specimens for biomarker analysis.

- ALK analysis (IHC and FISH) is applicable to both cytospins/ smears and cell blocks.

- ALK analysis of cytospins and liquid-based specimens requires different technical protocols to those used with cell blocks and histology.

\section{Reporting test results}

The aim of a molecular pathology report is to clearly communicate the results to clinicians in a language that is understandable to an oncologist or a fellow general pathologist. Any limitations and uncertainties in the test results should be explicitly communicated. Several published recommendations exist on how to format molecular test reports in general and NSCLC testing in particular; here, we will focus on integrated molecular reports written by the pathologist and important details that should be included in the respective sections of a test report.

\section{Integrated (combined) molecular reports written by the} pathologist

If molecular predictive testing is performed as an in-house reflex test, the results should preferably be reported as an addendum to the original report rather than being written as a stand-alone report. Ideally, the results and interpretations should be integrated as much as possible and written up by the pathologist.

\section{Preanalytical section}

Important parameters such as cold ischaemia time, fixative and fixation time need to be reported. If tumour cell enrichment is performed, the method of dissection must be denoted (eg, laser capture microdissection, manual microscopic, manual without microscopic, tissue core or whole section). The final content of tumour cells, expressed as percentage of total cells/nuclei, and the amount of DNA should be stated. Findings such as extensive necrosis, inflammation, pigmentation or borderline tumour cell content may be highlighted. An important control step is to inspect the last section after the material required for molecular analysis has been cut.

\section{Results section}

For clinically significant mutations, formal designations according to Human Genome Variation Society nomenclature should 
be presented together with a more colloquial locally acceptable nomenclature. Single nucleotide polymorphisms and variants of uncertain clinical significance need to be communicated. Although the International System for Cytogenetic Nomenclature can be used to describe chromosomal structural changes (eg, translocations and amplifications), many oncologists and pathologists are unfamiliar with this system and will also require terminology in common usage. For ISH-based tests, the number of cells analysed and the number and percentage of positive events should be stated. For multiplexed analyses or NGS results, a tabulated format is recommended. Inconclusive test results should be reported as such and the reason for the failure, if known, should be explained.

\section{Interpretation section (commentary)}

A statement of the probability of the cancer responding to or resisting specific targeted therapy should be included in this section.

\section{Technical section}

Sufficient technical information should be provided to enable another molecular pathologist to understand how testing was performed. Known limitations of tests need to be stated, and if positive and negative predictive values are published they need to be declared. The validation (IVD; CE; FDA) and accreditation (ISO; CAP) status of each test relays important information.

\section{Summary}

- Molecular test data reported by the pathologist and integrated as an addendum to original reports represent the optimal solution.

- Documentation of critical preanalytical factors, such as cold ischaemia and fixation time, represents an important first step in achieving better control of these factors.

- Communication of results according to established international consensus systems is necessary.

\section{Timing of testing and reporting}

There is increasing focus on reducing the interval between patients being referred to specialist care and the time treatment is started, as this may influence prognosis. ${ }^{38}$ Generally, clinicians expect to see a final molecular test report within five working days after the laboratory receives the specimen. After a confirmed diagnosis of NSCLC, transfer times between departments and the start of molecular testing must be kept to a minimum $(<24 \mathrm{~h})$. If tissue samples need to be sent to an outside laboratory for molecular testing, routines should be established so that unstained sections can be mailed within three working days after receiving the request or establishing final diagnosis if a reflex testing protocol exists. Here, we discuss relevant aspects of the timing of molecular tests and test reports.

\section{Preselection of suitable testing material in routine pathology} laboratory

Whenever a diagnosis of NSCLC is made from cytological, biopsy or surgical specimens, a section in the report should mention which slide or block is most suitable for ancillary molecular testing. A marked indication of the optimal tumour area on the selected slide and an estimate of tumour cell content should be given in the report.

Predefined panels and testing algorithms

A multidisciplinary group should make recommendations on a predictive test panel for NSCLC. A consensus decision for reflex upfront testing on initial diagnosis of NSCLC is optimal.
Parallel testing using multiplexing or NGS techniques is recommended. ${ }^{39}$

Early trigger point

Predictive molecular testing should be initiated when the first H\&E sections confirm probable NSCLC.

Digital pathology

Digital pathology holds great promise for reducing handling times and selecting the most appropriate tissue for analysis. The molecular pathologist can also decide if tumour cell enrichment techniques such as microdissection need to be used; if so, areas of interest can be indicated in the digitised image.

SOPs, including secretarial issues

In order to keep handling times for request forms, specimen requisitions and reporting to a minimum, it is very important to establish a SOP that includes the secretarial staff. Simple measures such as opening request forms addressed to individual doctors and scheduling a molecular pathologist for prioritised handling are essential.

\section{Electronic standard reports and LIS-HIS networks}

The utilisation of modern laboratory information systems (LIS) with built-in synoptic report generators for predictive molecular testing in NSCLC will significantly cut turnaround times. A good hospital information system (HIS) connected to all regional care providers and properly linked to the LIS should guarantee that results are transferred to the patient's physician immediately after the report is signed.

\section{Summary}

- Predefined test panels and algorithms including reflex and parallel testing, agreed by the multidisciplinary team, are recommended.

- Clear indication of the most suitable slide or block for predictive molecular testing and characterisation of tumour cell content should be included in the routine surgical pathology report.

- Utilisation of digital pathology to select suitable test material from digital archive and selection of area for tumour cell enrichment shorten handling time and costs.

- A SOP including secretarial handling of request forms and specimen requisitions needs to be established.

\section{External quality assessment/QC}

External quality assessment/QC at the European level

External quality assessment (EQA) is a systematic process for assessment of diagnostic and predictive tests, where a number of test samples are distributed to participating centres and subsequent test results are analysed. The goal is to achieve a high level of accuracy and reproducibility among different labs. Reaching this goal is vital to enable valid comparisons of global treatments, especially in the era of personalised therapy.

EQA programmes from various organisations have been in place since the beginning of molecular diagnostics. For predictive testing in NSCLC, reports have been published for $E G F R^{40-42}$ and $K R A S^{42}$ mutational analysis, and ALK. ${ }^{43} 44$ Guidelines for standardisation of EQA schemes have recently been introduced. $^{45}$

EQA/QC at the national level

The QuIP initiative ('Qualitätssicherungs-Initiative Pathologie') in Germany, Switzerland and Austria is an example of QC at the 
national level. To identify institutions capable of providing highquality molecular testing, QuIP organises EQAs procedures for different diagnostic applications. Until now, two ALK-QCs (based on ISH) had been enrolled in Germany, Switzerland and Austria: The first was performed at the end of 2012; 60.3\% $(32 / 53)$ of the participating institutes passed the EQA and were certified for ALK testing. ${ }^{43}$ The second ALK-QC took place at the beginning of 2014; in this programme, a total of $92.5 \%$ (37/40) of participating institutes passed the EQA, demonstrating a successful learning curve. EQAs might also help to gain experience in potential additional testing methods: ALK IHC was highlighted as an effective method for multicentre application, if carefully validated. ${ }^{39} 4344$

In June 2014, a joint agreement was signed by the German Society of Pathology, the Association of German Pathologists and the European Society of Pathology. Each organisation accepts the other's quality assessment process, and official documents (eg, certification) will be signed accordingly.

\section{Recommendations for QC at the national/European level}

- Sufficient performance in EQA schemes is crucial for comparison of global predictive biomarker studies.

- QC programmes (EQA) at the national and/or European level aim to provide a high level of accuracy and standardisation in predictive molecular testing.

- Pathology institutes should participate regularly in order to remain certified within EQA programmes.

- Only certified institutions should perform prognostic and predictive tests.

\section{Tumour heterogeneity}

Tumour heterogeneity is an issue one must be aware of, especially in the context of molecular diagnostics. Concerning renal clear cell carcinoma (RCC), this has been investigated in detail by Swanton and coworkers, showing that single RCCs harbour areas with different frequencies of mutations, suggesting the concept of main mutations (early development) and subclonal mutations (later development). This may provoke that biopsies do not represent a representative image of the tumour (the same can be true for metastasis). ${ }^{46} 47$

Concerning lung cancer, these aspects had been discussed for EGFR: time to disease progression and overall survival after gefitinib treatment were significantly shorter in those patients with EGFR heterogeneity. ${ }^{48}$

Concerning ALK, Camidge and coworkers showed different amounts of FISH-positive tumour cells within one tumour. However, they discussed this to be due to methodological rather than biological reasons. ${ }^{49}$

Furthermore, it was shown that so-called borderline cases with FISH positivity around the cut-off of $15 \%$ showed expression of the ALK-protein by IHC in nearly all tumour samples. ${ }^{22}$ To summarise, the role of intratumoural (spatial) heterogeneity is a growing concept that needs to be considered, especially when comparing biopsy and resections specimen, as well as primary site and metastasis. Upcoming NGS-based studies might help to further clarify these aspects and give answer to the question to what extent arbitrary results are due to biological and/or methodological reasons.

\section{CONCLUSIONS}

Pathobiological understanding, diagnostic accuracy and treatment options for NSCLC are rapidly evolving, leading to improvements in outcomes for many patients. This rapid evolution is driven by the new era of molecular targeted therapies with kinase inhibitors, and also by recent developments in the workflow of patient care, in particular:

- Better clinical diagnostics

- Refined sampling techniques

- Improved preanalytic measures of tissue handling

- Much more precise histological diagnoses, combined with

- New tissue-based or cytology-based molecular pathology assays

- Standardised reporting and

- Continuous external QC.

To bring together all these factors and optimise their effectiveness, multidisciplinary panels comprised of personnel experienced in different areas of cancer care are essential and may be key to further benefits. Thus, patients should be treated only in comprehensive cancer centres where these prerequisites are in place.

\section{Author affiliations}

${ }^{1}$ Institute of Pathology, Charité Universitätsmedizin Berlin, Berlin, Germany

${ }^{2}$ Institute of Pathology, University Hospital Basel, Basel, Switzerland

${ }^{3}$ Department of Respiratory Diseases, Maastricht University Medical Center, Maastricht, The Netherlands

${ }^{4}$ Respiratory Division, University Hospitals KU Leuven, Leuven, Belgium

${ }^{5}$ Department of Laboratory Medicine, Pathology, Örebro University Hospital, Örebro,

Sweden

${ }^{6}$ Department of Radiology, Hospital Universitario 12 de Octubre, Madrid, Spain

${ }^{7}$ Aberdeen University Medical School, Aberdeen, UK

${ }^{8}$ Academic Division of Thoracic Surgery, The Royal Brompton Hospital and Imperial

College, London, UK

${ }^{9}$ Laboratorio de Dianas Terapéuticas, Hospital Universitario HM Sanchinarro, Madrid, Spain

${ }^{10}$ Department of Pathology, VU University Medical Center, Amsterdam, The Netherlands

${ }^{11}$ Department of Thoracic and Vascular Surgery, Antwerp University Hospital, Antwerp, Belgium

Acknowledgements The authors would like to thank the following meeting participants: Professor Patrick Pauwels, (Department of Pathology, Antwerp University Hospital, Belgium), Dr Giulio Rossi (Anatomic Pathology Unit, Azienda Ospedaliero-Universitaria Policlinico of Modena, Italy), Professor Reinhard Büttner (Institute for Pathology, University Hospital Cologne, Germany), Professor Sakari Knuutila (Department of Pathology, University of Helsinki, Finland), Dr Ultan McDermott (Wellcome Trust Sanger Institute, Cambridge, UK), Dr Nicola Normanno (INT-Fondazione Pascale, Department of Experimental Oncology, Naples, Italy), Professor Frederique Penault-Llorca (Department of Pathology, Centre Jean-Perrin, Clermont-Ferrand, France), Professor Antonio Marchetti (Center of Predictive Molecular Medicine, Center of Excellence on Aging, University-Foundation, Chieti, Italy) and Mr Michael Gandy (University College London Advanced Diagnostics, University College Hospital, London, UK). Editorial assistance was provided by Ogilvy 4D, Oxford, UK.

Contributors MD was responsible for the conception and design of this work, drafting the sections of the manuscript which he authored/coauthored, revising these sections critically for important intellectual content and final approval of the whole manuscript. LB, A-MCD, CD, GE, RCG, KMK, EL, FL-R, ET, PEVS and MVL made substantial contributions to the conception and design of this work and were responsible for collaboratively drafting the sections of the manuscript which they authored/coauthored, revising those sections critically for important intellectual content and final approval of the whole manuscript. MD had full access to all content presented in the study and takes responsibility for the integrity and accuracy of the work.

Funding The meeting at which the concept for this manuscript was agreed was funded by Pfizer Pharma GmbH.

Competing interests $\mathrm{MD}$ : consultancy fees and honoraria from Pfizer; $\mathrm{LB}$ : honoraria from Abbott Mol., Inc. and from Pfizer; A-MCD: consultancy fees and/or honoraria (speakers' bureau) from Pfizer, Lilly, Roche, Boehringer Ingelheim, Novartis, Bristol-Myers Squibb and Merck Sharp and Dohme; GE: consultancy fees for scientific advisory boards from Pfizer and Qiagen; KMK: consultancy fees and honoraria (speakers' bureau) from Pfizer, Lilly, AstraZeneca, Roche, Boehringer Ingelheim and Novartis; EL: personal fees from Abbott Molecular, GlaxoSmithKline, Pfizer, Novartis, Covidien, Roche, Lilly Oncology, Boehringer Ingelheim, Medela, grants and personal fees from ScreenCell-he is also the founder of Informative Genomics, a blood-based molecular diagnostic company based in London; FL-R: honoraria from Pfizer, Novartis and Abbott; ET: consultancy fees and grants from Pfizer; MvL: consultancy fees and honoraria from Pfizer, Roche and Abbott. 
Provenance and peer review Not commissioned; externally peer reviewed.

Open Access This is an Open Access article distributed in accordance with the Creative Commons Attribution Non Commercial (CC BY-NC 4.0) license, which permits others to distribute, remix, adapt, build upon this work non-commercially, and license their derivative works on different terms, provided the original work is properly cited and the use is non-commercial. See: http://creativecommons.org/ licenses/by-nc/4.0/

\section{REFERENCES}

1 Soda M, Choi YL, Enomoto M, et al. Identification of the transforming EML4-ALK fusion gene in non-small-cell lung cancer. Nature 2007;448:561-6.

2 Thunnissen E, Bubendorf L, Dietel M, et al. EML4-ALK testing in non-small cell carcinomas of the lung: a review with recommendations. Virchows Arch 2012;461:245-57.

3 Davies KD, Le AT, Theodoro MF, et al. Identifying and targeting ROS1 gene fusions in non-small cell lung cancer. Clin Cancer Res 2012;18:4570-9.

4 Ou SH, Camidge DR, Riely G. Efficacy and safety of crizotinib in patients with advanced ROS1-rearranged non-small cell lung cancer (NSCLC). Ann Oncol 2013;24 (Suppl 9):ix43.

5 Dietel $\mathrm{M}$, Johrens $\mathrm{K}$, Laffert $\mathrm{M}$, et al. Predictive molecular pathology and its role in targeted cancer therapy: a review focussing on clinical relevance. Cancer Gene Ther 2013;20:211-21.

6 Travis WD, Brambilla E, Noguchi M, et al. International Association for the Study of Lung Cancer/American Thoracic Society/European Respiratory Society: international multidisciplinary classification of lung adenocarcinoma: executive summary. Proc Am Thorac Soc 2011;8:381-5.

7 McCloskey P, Balduyck B, Van Schil PE, et al. Radical treatment of non-small cell lung cancer during the last 5 years. Eur J Cancer 2013;49:1555-64.

8 Annema JT, van Meerbeeck JP, Rintoul RC, et al. Mediastinoscopy vs endosonography for mediastinal nodal staging of lung cancer: a randomized trial. JAMA 2010;304:2245-52.

9 De Leyn P, Dooms C, Kuzdzal J, et al. Revised ESTS guidelines for preoperative mediastinal lymph node staging for non-small-cell lung cancer. Eur J Cardiothorac Surg 2014;45:787-98.

10 Hetzel J, Eberhardt R, Herth FJ, et al. Cryobiopsy increases the diagnostic yield of endobronchial biopsy: a multicentre trial. Eur Respir J 2012;39:685-90.

11 Du Rand IA, Blaikley J, Booton R, et al. British Thoracic Society guideline for diagnostic flexible bronchoscopy in adults: accredited by NICE. Thorax 2013;68 (Suppl 1):i1-44.

12 Popovich J Jr, Kvale PA, Eichenhorn MS, et al. Diagnostic accuracy of multiple biopsies from flexible fiberoptic bronchoscopy. A comparison of central versus peripheral carcinoma. Am Rev Respir Dis 1982;125:521-3.

13 Dooms C, Muylle I, Yserbyt J, et al. Endobronchial ultrasound in the management of non small cell lung cancer. Eur Respir Rev 2013;22:169-77.

14 Wu CC, Maher MM, Shepard JA. CT-guided percutaneous needle biopsy of the chest: preprocedural evaluation and technique. AJR Am J Roentgenol 2011;196: W511-14.

15 Kothary N, Lock L, Sze DY, et al. Computed tomography-guided percutaneous needle biopsy of pulmonary nodules: impact of nodule size on diagnostic accuracy. Clin Lung Cancer 2009;10:360-3.

16 Westra WH, Hruban RH, Phelps TH, et al. Surgical pathology dissection: an illustrated guide. 2nd edn. New York, NY: Springer-Verlag, 2003.

17 Wittekind C, Meyer HJ. TNM classification of malignant tumours. 7th edn. New York, NY: Wiley-Blackwell, 2011:336.

18 Kerr KM, Bubendorf L, Edelman MJ, et al. Second ESMO consensus conference on lung cancer: pathology and molecular biomarkers for non-small-cell lung cancer. Ann Oncol 2014;25:1681-90.

19 Tsao MH, Hirsch FR, Yatabe Y, eds. IASLC atlas of ALK testing in lung cancer. Aurora, CO: International Association for the Study of Lung Cancer, 2013.

20 Bussolati G, Annaratone L, Medico E, et al. Formalin fixation at low temperature better preserves nucleic acid integrity. PLOS ONE 2011;6:e21043.

21 Muller BM, Brase JC, Haufe F, et al. Comparison of the RNA-based EndoPredict multigene test between core biopsies and corresponding surgical breast cancer sections. J Clin Pathol 2012;65:660-2.

22 von Laffert $M$, Warth $A$, Penzel $R$, et al. Multicenter immunohistochemical ALK-testing of non-small-cell lung cancer shows high concordance after harmonization of techniques and interpretation criteria. J Thorac Oncol 2014;9:1685-92.

23 Conde $E$, Angulo $B$, Izquierdo $E$, et al. Lung adenocarcinoma in the era of targeted therapies: histological classification, sample prioritization, and predictive biomarkers. Clin Transl Oncol 2013;15:503-8.

24 Lee HJ, Seol HS, Kim JY, et al. ROS1 receptor tyrosine kinase, a druggable target, is frequently overexpressed in non-small cell lung carcinomas via genetic and epigenetic mechanisms. Ann Surg Oncol 2013;20:200-8.
25 Sekine I, Yamamoto N, Nishio K, et al. Emerging ethnic differences in lung cancer therapy. Br J Cancer 2008;99:1757-62.

26 Conde $E$, Suarez-Gauthier $A$, Benito $A$, et al. Accurate identification of ALK positive lung carcinoma patients: novel FDA-cleared automated fluorescence in situ hybridization scanning system and ultrasensitive immunohistochemistry. PLOS ONE 2014;9:e107200.

27 Fu S, Wang F, Shao Q, et al. Detection of EML4-ALK fusion gene in Chinese non-small cell lung cancer by using a sensitive quantitative real-time reverse transcriptase PCR technique. Diagn Mol Pathol 2015;23:245-54.

28 Li T, Maus MK, Desai SJ, et al. Large-scale screening and molecular characterization of EML4-ALK fusion variants in archival non-small-cell lung cancer tumor specimens using quantitative reverse transcription polymerase chain reaction assays. J Thorac Oncol 2014:9:18-25.

29 Lira ME, Choi YL, Lim SM, et al. A single-tube multiplexed assay for detecting ALK, ROS1, and RET fusions in lung cancer. J Mol Diagn 2014;16:229-43.

30 Zhang NN, Liu YT, Ma L, et al. The molecular detection and clinical significance of ALK rearrangement in selected advanced non-small cell lung cancer: ALK expression provides insights into ALK targeted therapy. PLoS ONE 2014;9:e84501.

31 Pan Y, Zhang Y, Li Y, et al. ALK, ROS1 and RET fusions in 1139 lung adenocarcinomas: a comprehensive study of common and fusion patternspecific clinicopathologic, histologic and cytologic features. Lung Cancer 2014;84:121-6.

32 Wang $R$, Pan $Y, L i C$, et al. The use of quantitative real-time reverse transcriptase $P C R$ for $5^{\prime}$ and $3^{\prime}$ portions of ALK transcripts to detect ALK rearrangements in lung cancers. Clin Cancer Res 2012;18:4725-32.

33 Huang D, Kim DW, Kotsakis A, et al. Multiplexed deep sequencing analysis of ALK kinase domain identifies resistance mutations in relapsed patients following crizotinib treatment. Genomics 2013;102:157-62.

34 Shaw AT, Kim DW, Nakagawa K, et al. Crizotinib versus chemotherapy in advanced ALK-positive lung cancer. N Engl I Med 2013;368:2385-94.

35 Knoepp SM, Roh MH. Ancillary techniques on direct-smear aspirate slides: a significant evolution for cytopathology techniques. Cancer Cytopathol 2013;121:120-8.

36 Savic $S$, Bubendorf $L$. Role of fluorescence in situ hybridization in lung cancer cytology. Acta Cytol 2012;56:611-21.

37 Savic S, Bode B, Diebold J, et al. Detection of ALK-positive non-small-cell lung cancers on cytological specimens: high accuracy of immunocytochemistry with the 5A4 clone. J Thorac Oncol 2013;8:1004-11.

38 Kassahn KS, Scott HS, Caramins MC. Integrating massively parallel sequencing into diagnostic workflows and managing the annotation and clinical interpretation challenge. Hum Mutat 2014;35:413-23.

39 Lindeman NI, Cagle PT, Beasley MB, et al. Molecular testing guideline for selection of lung cancer patients for EGFR and ALK tyrosine kinase inhibitors: guideline from the College of American Pathologists, International Association for the Study of Lung Cancer, and Association for Molecular Pathology. Arch Pathol Lab Med 2013;137:828-60.

40 Deans ZC, Bilbe N, O'Sullivan B, et al. Improvement in the quality of molecular analysis of EGFR in non-small-cell lung cancer detected by three rounds of external quality assessment. J Clin Pathol 2013;66:319-25.

41 Normanno N, Pinto C, Taddei G, et al. Results of the First Italian External Quality Assurance Scheme for somatic EGFR mutation testing in non-small-cell lung cancer. J Thorac Oncol 2013;8:773-8.

42 Thunnissen E, Bovee JV, Bruinsma H, et al. EGFR and KRAS quality assurance schemes in pathology: generating normative data for molecular predictive marker analysis in targeted therapy. J Clin Pathol 2011;64:884-92.

43 von Laffert M, Penzel R, Schirmacher P, et al. Multicenter ALK testing in non-small-cell lung cancer: results of a round robin test. J Thorac Oncol 2014;9:1464-9.

44 von Laffert $M$, Warth A, Penzel R, et al. Anaplastic lymphoma kinase (ALK) gene rearrangement in non-small cell lung cancer (NSCLC): results of a multi-centre ALK-testing. Lung Cancer 2013;81:200-6.

45 van Krieken JH, Normanno N, Blackhall F, et al. Guideline on the requirements of external quality assessment programs in molecular pathology. Virchows Arch 2013;462:27-37.

46 Gerlinger M, Horswell S, Larkin J, et al. Genomic architecture and evolution of clear cell renal cell carcinomas defined by multiregion sequencing. Nat Genet 2014;46:225-33.

47 Swanton C. Intratumor heterogeneity: evolution through space and time. Cancer Res 2012;72:4875-82.

48 Taniguchi K, Okami J, Kodama K, et al. Intratumor heterogeneity of epidermal growth factor receptor mutations in lung cancer and its correlation to the response to gefitinib. Cancer Sci 2008:99:929-35.

49 Camidge DR, Skokan M, Kiatsimkul P, et al. Native and rearranged ALK copy number and rearranged cell count in non-small cell lung cancer: implications for ALK inhibitor therapy. Cancer 2013;119:3968-75. 